\title{
Photon statistics of quantum light on scattering from rotating ground glass
}

\author{
Sheng-Wen Li, ${ }^{1,2} \mathrm{Fu} \mathrm{Li},{ }^{2}$ Tao Peng, ${ }^{2}$ and G. S. Agarwal ${ }^{2}$ \\ ${ }^{1}$ Center for quantum technology research, School of Physics, \\ Beijing Institute of Technology, Beijing 100081, People's Republic of China \\ ${ }^{2}$ Texas A\&M University, College Station, Texas 77843, USA
}

(Dated: November 21, 2019)

\begin{abstract}
When a laser beam passes through a rotating ground glass (RGG), the scattered light exhibits thermal statistics. This is extensively used in speckle imaging. This scattering process has not been addressed in photon picture and is especially relevant if non-classical light is scattered by the RGG. We develop the photon picture for the scattering process using the Bose statistics for distributing $N$ photons in $M$ pixels. We obtain analytical form for the $P$-distribution of the output field in terms of the $P$-distribution of the input field. In particular we obtain a general relation for the $n$-th order correlation function of the scattered light, i.e., $g_{\text {out }}^{(n)} \simeq n ! g_{\text {in }}^{(n)}$, which holds for any order- $n$ and for arbitrary input states. This result immediately recovers the classical transformation of coherent light to pseudo-thermal light by RGG.
\end{abstract}

\section{INTRODUCTION}

Laser light usually carries a (super-)Poisson photon statistics [1-4], but when a laser beam passes through a rotating ground glass (RGG), the scattered light exhibits a thermal statistics ${ }^{1}$. This is known as the "pseudo-thermal" light, which has been well verified in experiments [5-10], and widely used for ghost imaging [11-13], sub-wavelength imaging and lithography $[14,15]$, as well as some fundamental studies [16-18].

It is quite interesting that the input Poisson statistics can be changed to be the thermal one in such a simple way. Usually this is understood in classical theory $[19,20]$ : the light field $E$ collected at the photon detector is the superposition of the sub-fields $\tilde{E}_{i}$ propagated from different positions of the RGG. Due to the rotation of the disc, the phases and amplitudes of these sub-fields $\tilde{E}_{i}$ are varying with time randomly. According to the central limit theorem, their summation $E$, as a random variable, exhibits a Gaussian distribution $\left[P(E) \sim \exp \left(-\beta|E|^{2}\right)\right]$, and thus the intensity $\left(I \sim|E|^{2}\right)$ exhibits a negative-exponential distribution, i.e., the thermal distribution [19-22].

In principle, the central limit theorem requires the subsources $\tilde{E}_{i}$ should be infinitely many point sources. Experimentalaly, the number of sub-sources is limitted by the granules within the light spot on the ground glass; thus the pseudothermal light generated in experiments shows some deviation from the ideal thermal distribution [23-25].

Rigorously speaking, the continuous distribution $P(I)$ for the light intensity is a classical treatment, but not exactly equivalent with the distribution $P_{n}$ for the quantized photon numbers, especially in the few-photon regime. Moreover, if the input light is a non-classical state, the above classical interpretation does not apply, and clearly the output light is generally no longer the thermal one.

\footnotetext{
1 Throughout this paper, we focus on the "thermal light" with a single frequency, namely, only one optical mode is in the thermal state, and all the other field modes are in the vacuum state.
}

In this sense, although the transformation of coherent light on a diffusing glass plate has been extensively investigated [6$10,19,20]$, and achieved wide applications in speckle imaging [11-13, 24-26], most time it was based on the above classical understanding rather than a fully-quantized photon picture. In particular, if the input light intensity is at single photon level, or is scattered by very few diffusers, the validity of above classical theory needs to be checked more carefully.

In this paper, we develop a quantum framework to study the full photon number statistics of such light scattered from general input states. The basic picture is, when $\mathbb{N}_{P}$ photons are scattered randomly by the RGG, the photon number received by a small area fluctuates stochastically. Therefore, the photon number statistics is obtained by counting the combinations how these photons are randomly distributed on the scattered light pattern, and that naturally gives the thermal statistics.

Then for arbitrary input states, the scattered photon statistics also can be well obtained by taking the input statistics into account. Moreover, we prove that the scattered $g_{\text {out }}^{(2)}$ is always twice of the input $g_{\mathrm{out}}^{(2)} \simeq 2 g_{\mathrm{in}}^{(2)}$ for any input states, including non-classical states. Besides, we also obtain the $P$-function of the scattered light in the many-diffuser limit, and that further gives the relation $g_{\text {out }}^{(n)} \simeq n ! g_{\text {in }}^{(n)}$ for high-order correlations of any order- $n$ and any input.

The paper is arranged as follows. In Sec. II, we discuss the basic generation mechanism of the pseudo-thermal light. In Sec. III, we obtain the scattered $g^{(2)}$-correlation for arbitrary input state. In Sec. IV, we discuss the scattered $P$-function and high-order correlations. The summary is drawn in Sec. V.

\section{THE PSEUDO-THERMAL LIGHT GENERATION FROM PHOTON SCATTERING}

We first briefly review the basic photon counting process [Fig. 1(a)]. Considering a steady light flux coming into the photon detector (PD), in each exposure period, a quantum projective measurement is made on the light intensity. The exposure time must be much shorter than the light coherence time, otherwise the measured result is indeed a time average, which corresponds to classical treatments [21, 22]. In the ide- 
alistic case, the data $\left\{n_{t}\right\}$ of many exposure frames should be a stochastic series of integers due to the quantized feature of photons, as well as the intrinsic randomness of quantum measurements [27-30]. This can be regarded as multiple repetitive measurements on the same light state. Counting $\left\{n_{t}\right\}$ gives the photon number distribution $P_{n}$ of the incoming light.

Therefore, the pseudo-thermal light generation can be understood as follows. Considering a laser beam divergently scattered by a RGG, we suppose all the scattered light is collected by a pixel lattice composed of $M(\gg 1)$ independent PDs. In each exposure, this pixel lattice takes a photo frame of the whole scattered light, which appears as a light pattern randomly distributed in spatial domain [Fig. 1(b)]. When the ground glass is moving, this light pattern varies with time like a "zoetrope". Thus, when focusing on a small area on the light pattern, its intensity fluctuates with time stochastically, which is similar like the above quantum measurement data. This is just how the pseudo-thermal light is obtained.

Thus, the photon statistics on a certain pixel can be calculated by counting how the input photons are distributed on the whole pixel lattice, as shown below.

\section{Fock input:}

We first consider a simple case that in each frame, the incoming light always contains exactly $\mathrm{N}_{\mathrm{P}}$ photons (Fock input). Due to the stochastic scattering, these $\mathrm{N}_{\mathrm{P}}$ photons would be randomly distributed to all the $M$ pixels. Denoting $n_{\mathbf{x}, t}$ as the photon number recorded at pixel-x in frame- $t$, all possible pattern configurations $\left\{n_{\mathbf{x}}\right\}$ may appear (assuming with equal probabilities). Remember all $n_{\mathbf{x}}$ are integers $\left(0 \leq n_{\mathbf{x}} \leq \mathrm{N}_{\mathrm{P}}\right)$, and they must satisfy the constraint of energy conservation $\sum_{\mathrm{x}} n_{\mathrm{x}}=\mathrm{N}_{\mathrm{P}}$. Thus, since photons are identical bosons, the total number of such combinations is given by $[31,32]^{2}$

$$
Z:=\left(\begin{array}{c}
\mathrm{N}_{\mathrm{P}}+M-1 \\
M-1
\end{array}\right)=\frac{\left(\mathrm{N}_{\mathrm{P}}+M-1\right) !}{(M-1) ! \mathrm{N}_{\mathrm{P}} !} .
$$

Further, we count the probability that $n$ photons are received by a single pixel. That is equivalent as counting the combinations how $\mathrm{N}_{\mathrm{P}}-n$ photons are distributed on the rest $M-1$ pixels, which gives (for $M \geq 2$ )

$$
P_{n}^{\left(\mathrm{N}_{\mathrm{P}}\right)}= \begin{cases}\frac{1}{Z}\left(\mathrm{~N}_{\mathrm{P}}-n+M-2\right), & 0 \leq n \leq \mathrm{N}_{\mathrm{P}} \\ 0, & n>\mathrm{N}_{\mathrm{P}}\end{cases}
$$

This is just the scattered photon statistics on one pixel when the input light carries $N_{P}$ photons in each frame.

In the regime $n \ll N_{\mathrm{P}}$, the above probability gives

$$
\frac{P_{n+1}^{\left(\mathrm{N}_{\mathrm{P}}\right)}}{P_{n}^{\left(\mathrm{N}_{\mathrm{P}}\right)}}=\frac{1}{1+\frac{M-2}{\mathrm{~N}_{\mathrm{P}}-n}} \simeq \frac{1}{1+M / \mathrm{N}_{\mathrm{P}}},
$$

\footnotetext{
2 This is equivalent as counting the combinations how $\mathrm{N}_{\mathrm{P}}+M$ identical balls are put into $M$ different boxes, with zero reception allowed.
}
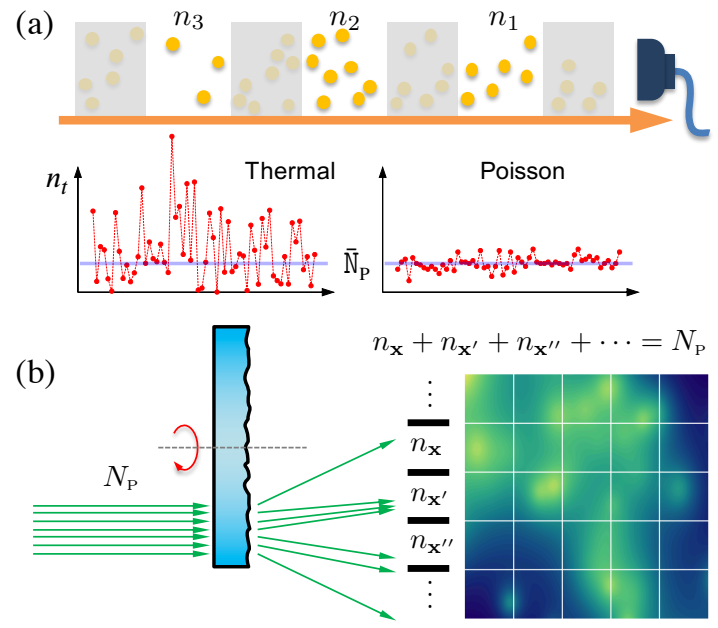

Figure 1. (a) Demonstration for the photon counting process. (b) The light beam is scattered divergently by the RGG and generates a random light pattern.

which is a constant and less than 1 (when $M \gg 1$ ). That means, the statistics received on this pixel well exhibits the thermal distribution. In the regime $n \sim \mathrm{N}_{\mathrm{P}}$, the deviation from the ideal thermal distribution gradually grows larger, but this difference is not easy to be observed in practical experiments (Fig. 2).

Therefore, when focusing on the photon statistics received on a local area, the conservation constraint of the total photon number naturally leads to the thermal statistics. Indeed this is quite similar with the mechanism how the canonical ensemble with the thermal statistics emerges as a subsystem inside a bigger micro-canonical one in statistical physics [31, 32].

\section{Poisson input:}

The incoming light could also have certain distribution $\mathcal{P}_{\text {in }}(\mathrm{N})$ but not exactly $\mathrm{N}_{\mathrm{P}}$ photons. In this case, taking the input statistics into account, the scattered statistics is

$$
P_{n}=\sum_{\mathrm{N}} \mathcal{P}_{\text {in }}(\mathrm{N}) \cdot P_{n}^{(\mathrm{N})},
$$

where $P_{n}^{(\mathrm{N})}$ is the result from the scattering of $\mathrm{N}$ photons [Eq. (2)].

For a laser input, the input statistics $\mathcal{P}_{\text {in }}(\mathrm{N})$ is approximately a Poisson distribution, which is narrowly distributed around its mean value $\overline{\mathrm{N}}_{\mathrm{P}}$ with a relatively small variance $\left(\sqrt{\left\langle\delta n^{2}\right\rangle} /\langle n\rangle=1 / \sqrt{\mathbb{N}_{\mathrm{P}}}\right)$. Therefore, in the above summation (4), only the terms around $\mathrm{N} \simeq \overline{\mathrm{N}}_{\mathrm{P}}$ contribute significantly. As a result, approximately the output photon statistics is also a thermal one, which satisfies

$$
\frac{P_{n+1}}{P_{n}} \simeq \frac{1}{1+M / \overline{\mathrm{N}}_{\mathrm{P}}} .
$$

For the above Fock and Poisson input cases, the exact output distributions $P_{n}$ are numerically shown in Fig. 2(a,b), and 

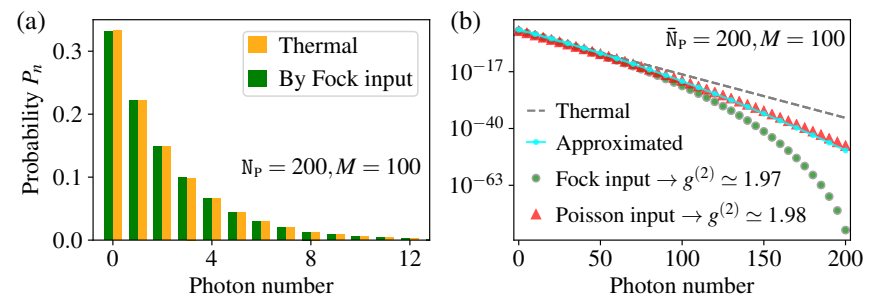

Figure 2. (a) The scattered photon distribution $P_{n}$ on one pixel from Fock input (left green), comparing with the thermal one (right yellow) with the same mean photon number. (b) The scattered photon distribution (in $\log$ scale) from the Fock and Poisson input $\left(\overline{\mathrm{N}}_{\mathrm{P}}=200\right)$ comparing with the thermal distribution (dashed gray). The dotted cyan line is the approximated result (7).

they are both quite close to the thermal distribution in the regime $n \ll \overline{\mathrm{N}}_{\mathrm{P}}$. When the photon number $n$ is large, both of them deviate from the thermal one.

\section{Non-thermal correction:}

Here we give an approximated distribution taking account the above non-thermal correction. We expand the above iterative relation (3) as follows

$$
\begin{aligned}
& \ln P_{n+1}^{\left(\mathrm{N}_{\mathrm{P}}\right)}-\ln P_{n}^{\left(\mathrm{N}_{\mathrm{P}}\right)}=\ln \frac{1}{1+\frac{M-2}{\mathrm{~N}_{\mathrm{P}}-n}} \\
& \quad \simeq \ln \frac{1}{1+\frac{M-2}{\mathrm{~N}_{\mathrm{P}}}}-n \cdot \frac{M-2}{\mathrm{~N}_{\mathrm{P}}\left(\mathrm{N}_{\mathrm{P}}+M-2\right)}+o\left(n^{2}\right) .
\end{aligned}
$$

Omitting the high order terms $o\left(n^{2}\right)$, the summation over $n$ on both sides gives the following approximated distribution [the dotted cyan line in Fig. 2(b)]

$$
\begin{aligned}
P_{n}^{\left(\mathrm{N}_{\mathrm{P}}\right)} & =P_{0} e^{-\beta_{0} n-\beta_{\mathrm{c}}\left(n^{2}-n\right)}, \\
\beta_{0} & =\ln \left(1+\frac{M-2}{\mathrm{~N}_{\mathrm{P}}}\right), \quad \beta_{\mathrm{c}}=\frac{1}{2} \frac{M-2}{\mathrm{~N}_{\mathrm{P}}\left(\mathrm{N}_{\mathrm{P}}+M-2\right)},
\end{aligned}
$$

where $P_{0}$ is a normalization constant. A quadratic correction appears in the exponential factor, and the correction $\beta_{\mathrm{c}}$ is negligible for strong input $\mathrm{N}_{\mathrm{P}}$. Indeed, this is a typical noncanonical feature resulted from finite system sizes or coupling strengths [33, 34].

At last we remark that the "pixels" in the above discussions do not corresponds to realistic detector pixels directly. The sizes of such pixels are determined by the spatial correlation length of the scattered light pattern, so they can be regarded as independent fluctuation units. And their total number $M$ is roughly determined by the granules within the light spot on the RGG, which is usually a very large number in practice.

\section{THE SCATTERED STATISTICS FROM NON-CLASSICAL LIGHT}

If the input light is a non-classical state, the standard classical theory does not apply $[19,20]$. In this case, generally the scattered photon statistics is no longer a thermal one. As long as the corresponding input $\mathcal{P}_{\text {in }}(\mathrm{N})$ is taken, the general result for the scattered photon statistics $P_{n}$ can be obtained [Eq. (4)].

Comparing with the distribution profile $P_{n}$, the $g^{(2)}{ }_{-}$ correlation is indeed a more precise indicator to characterize the photon statistics [3,4]. For example, considering averagely $\overline{\mathrm{N}}_{\mathrm{P}}=8$ photons scattered onto $M=8$ fluctuating units, although the scattered $P_{n}$ from both Fock and Poisson inputs look quite close to the thermal one [Fig. 3(a)], indeed they give $g_{\text {out }}^{(2)} \simeq 1.556$ (Fock input) and $g_{\text {out }}^{(2)} \simeq 1.778$ (Poisson input), which are both significantly different from the thermal result $g_{\text {th }}^{(2)}=2$.

For arbitrary input states, the scattered $g^{(2)}$-correlation can be calculated from Eq. (4). The mean photon number of the scattered light is $\langle n\rangle=\langle\mathrm{N}\rangle_{\text {in }} / M$, and the mean square is:

$$
\begin{aligned}
\left\langle n^{2}\right\rangle & =\sum_{\mathrm{N}, n} \mathcal{P}_{\text {in }}(\mathrm{N}) \cdot n^{2} P_{n}^{(\mathrm{N})}=\sum_{\mathrm{N}} \mathcal{P}_{\text {in }}(\mathrm{N}) \frac{\mathrm{N}(2 \mathrm{~N}+M-1)}{M(M+1)} \\
& =\frac{2\left\langle\mathrm{~N}^{2}\right\rangle_{\text {in }}}{M(M+1)}+\frac{\langle\mathrm{N}\rangle_{\text {in }}(M-1)}{M(M+1)} .
\end{aligned}
$$

Then after simple calculations, the scattered $g_{\text {out }}^{(2)}=\left(\left\langle n^{2}\right\rangle-\right.$ $\langle n\rangle) /\langle n\rangle^{2}$ can be represented by the input $g_{\text {in }}^{(2)}$ :

$$
g_{\text {out }}^{(2)}=\frac{2\left(\left\langle\mathrm{~N}^{2}\right\rangle_{\text {in }}-\langle\mathrm{N}\rangle_{\text {in }}\right) M}{\langle\mathrm{~N}\rangle_{\text {in }}^{2}(M+1)}=2 g_{\text {in }}^{(2)} \cdot \frac{M}{M+1} .
$$

In practice, the RGG usually has a large number of diffusers ( $M \gg 1$ ), which gives $g_{\text {out }}^{(2)} \simeq 2 g_{\text {in }}^{(2)}$. It is worth noticing this is a general result for arbitrary input statistics, including nonclassical light.

Therefore, a laser input with Poisson statistics always leads to the thermal result $g_{\text {out }}^{(2)} \simeq 2$, which is irrelevant of the input intensity, even at the single-photon level $\overline{\mathrm{N}}_{\mathrm{P}}=1$.

On contrary, the Fock input $\left|\mathrm{N}_{\mathrm{P}}\right\rangle$ has $g_{\mathrm{in}}^{(2)}=1-\mathrm{N}_{\mathrm{P}}^{-1} \in$ $[0,1)$, and that makes the scattered $g_{\text {out }}^{(2)} \simeq 2 g_{\text {in }}^{(2)}$ increase with the input photon number $\mathrm{N}_{\mathrm{P}}$ [Fig. 3(c)]. In particular, the single-photon input leads to a scattered state $\hat{\rho}_{\text {out }}=$ $\left(1-\frac{1}{M}\right)|0\rangle\left\langle 0\left|+\frac{1}{M}\right| 1\right\rangle\langle 1|$ with $g_{\text {out }}^{(2)}=0$.

Thus, the quantum features of non-classical states appear more significant in the few-photon regime. And it is also worth to notice that the Poisson input plays better in producing the thermal statistics than the Fock input, since it always guarantees $g_{\text {out }}^{(2)} \simeq 2$ irrelevant of the input intensity.

With the increase of the total number $M$ of the fluctuating units, the scattered $g_{\text {out }}^{(2)}$ increases and converges to $g_{\text {out }}^{(2)} \simeq$ $2 g_{\text {in }}^{(2)}$ [Fig. 3(b)]. And this just corresponds to the condition of infinite diffusers required by the central limit theorem in classical theory.

By utilizing the squeezed light, a non-classical input state with sub-Poisson statistics can be realized in experiments $[4,35,36]$. In Fig. 3(d) we show the scattered $g_{\text {out }}^{(2)}$ from the squeezed light calculated from Eq. (4). The input statistics $\mathcal{P}_{\text {in }}(\mathrm{N})$ comes from the squeezed state $|\alpha, \xi\rangle=\hat{D}_{\alpha} \hat{S}_{\xi}|0\rangle$, where $\hat{S}_{\xi}:=\exp \left[\frac{1}{2}\left(\xi^{*} \hat{a}^{2}-\xi \hat{a}^{\dagger 2}\right)\right], \hat{D}_{\alpha}:=\exp \left[\alpha \hat{a}^{\dagger}-\alpha^{*} \hat{a}\right]$ 

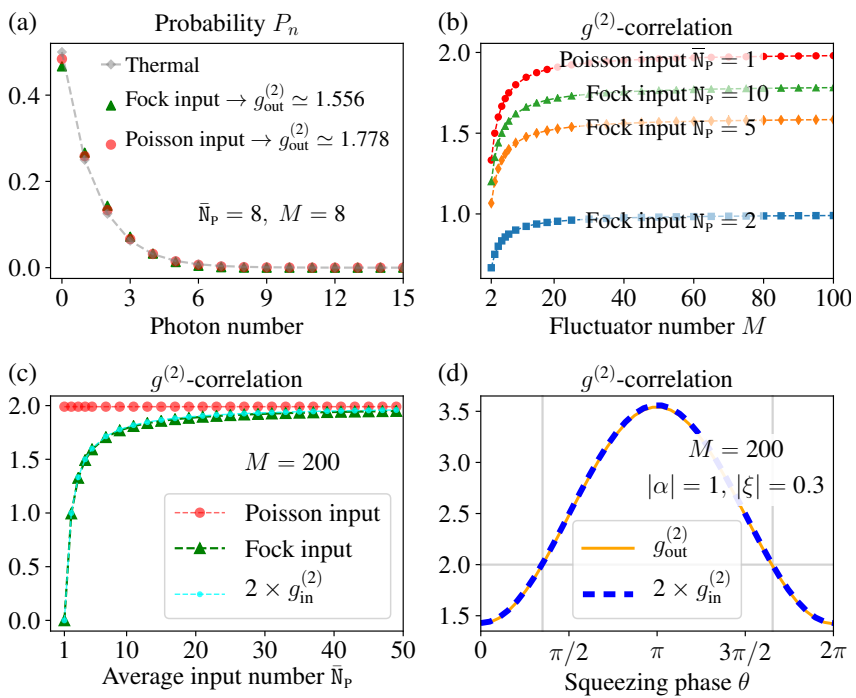

Figure 3. (a) The scattered distribution $P_{n}$ from Fock and Poisson input $\left(\overline{\mathrm{N}}_{\mathrm{P}}=8, M=8\right)$. (b) The scattered $g_{\text {out }}^{(2)}$ from Fock $\left(\mathrm{N}_{\mathrm{P}}=\right.$ $2,5,10)$ and Poisson input $\left(\overline{\mathrm{N}}_{\mathrm{P}}=1\right)$ changing with fluctuator number $M$. (c) The scattered $g_{\text {out }}^{(2)}$ changing with the input photon number (given $M=200$ ), comparing with the input $g_{\text {in }}^{(2)}$ of Fock state. (d) The scattered $g_{\text {out }}^{(2)}$ from squeezed input changing with the squeezing phase $\theta$, comparing with the input $g_{\text {in }}^{(2)}$.

are the squeezing and displacement operators, with $\xi=r e^{i \theta}$ $(r \geq 0)$ as the squeezing parameter $[4,36]$.

For different squeezing phases $\theta$, the mean photon number keeps to be $\overline{\mathrm{N}}_{\mathrm{P}}=|\alpha|^{2}+\sinh ^{2} r$, while the input statistics gives sub-Poisson $\left(g_{\text {in }}^{(2)}<1\right)$ or super-Poisson $\left(g_{\text {in }}^{(2)}>1\right)$ distributions. In the whole regime the above relation $g_{\text {out }}^{(2)} \simeq$ $2 g_{\text {in }}^{(2)}$ well applies.

Notice that the output light can be used as the input light for further scattering through another RGG, then that gives $g_{\text {out-2 }}^{(2)} \simeq 2 g_{\text {out-1 }}^{(2)} \simeq 2^{2} g_{\text {in }}^{(2)}$. Clearly, after the hierarchy scattering through $k$ RGGs, the final output light has $g_{\text {out } k}^{(2)} \simeq 2^{k} g_{\text {in }}^{(2)}$. In particular, if the original input is laser, the final output gives $g_{\text {out }-k}^{(2)} \simeq 2^{k}[19,20,37]$.

Besides, similarly like Eq. (8), the 3rd order expectation $\left\langle n^{3}\right\rangle$ and $g_{\text {out }}^{(3)}$ also can be obtained, i.e.,

$$
\begin{aligned}
\left\langle n^{3}\right\rangle & =\frac{6\left\langle\mathrm{~N}^{3}\right\rangle_{\text {in }}+6(M-1)\left\langle\mathrm{N}^{2}\right\rangle_{\text {in }}+\left(M^{2}-3 M+2\right)\langle\mathrm{N}\rangle_{\text {in }}}{M(M+1)(M+2)}, \\
g_{\text {out }}^{(3)} & =\frac{\left\langle\hat{a}^{\dagger 3} \hat{a}^{3}\right\rangle_{\text {out }}}{\left\langle\hat{a}^{\dagger} \hat{a}\right\rangle_{\text {out }}^{3}}=6 g_{\text {in }}^{(3)} \cdot \frac{M^{2}}{M^{2}+3 M+2},
\end{aligned}
$$

which gives $g_{\text {out }}^{(3)} \simeq 6 g_{\text {in }}^{(3)}$ for large $M$. But the calculation of higher orders becomes more and more troublesome. In the following, with the help of the $P$-function of the scattered light, we can prove $g_{\text {out }}^{(n)} \simeq n ! g_{\text {in }}^{(n)}$ for any order- $n$ and any input state.

\section{THE $P$-FUNCTION OF THE SCATTERED LIGHT AND THE HIGH-ORDER CORRELATIONS}

The non-classical properties of a light state can be more clearly characterized by its $P$-function $[3,4,38]$, and here we show the $P$-function of the scattered light and its high-order correlations. As seen above, the Poisson input plays better in producing the thermal statistics than the Fock input, since it always leads to the thermal result $g_{\text {out }}^{(2)} \simeq 2$ in spite of the input intensity (in the many-diffuser limit $M \rightarrow \infty$ ). Thus, approximately this can be written as the following mapping relation:

$$
\begin{gathered}
\hat{\rho}_{\text {in }}=\left|\alpha_{0}\right\rangle\left\langle\alpha_{0}\left|\quad \rightarrow \hat{\rho}_{\text {out }}=\sum \frac{\overline{\mathrm{N}}_{T}^{m}}{\left(1+\overline{\mathrm{N}}_{T}\right)^{n+1}}\right| n\right\rangle\langle n|, \\
P_{\text {in }}(\alpha)=\delta^{(2)}\left(\alpha-\alpha_{0}\right) \rightarrow P_{\text {out }}(\alpha)=\frac{1}{\pi \overline{\mathrm{N}}_{T}} \exp \left(-\frac{|\alpha|^{2}}{\overline{\mathrm{N}}_{T}}\right) .
\end{gathered}
$$

Here $\left|\alpha_{0}\right|^{2}$ and $\overline{\mathrm{N}}_{T}=\left|\alpha_{0}\right|^{2} / M$ are the mean photon numbers of the input and output statistics.

This input-output relation can be written as a linear functional transformation $P_{\text {out }}(\alpha)=\mathscr{F}_{\alpha}\left[P_{\text {in }}(\alpha)\right]$. Then for a general input state $P_{\text {in }}(\alpha)=\int d^{2} \varsigma P_{\text {in }}(\varsigma) \delta^{(2)}(\alpha-\varsigma)$, the output $P$-function is

$$
\begin{aligned}
P_{\text {out }}(\alpha) & =\mathscr{F}_{\alpha}\left[P_{\text {in }}(\alpha)\right]=\int d^{2} \varsigma P_{\text {in }}(\varsigma) \mathscr{F}_{\alpha}\left[\delta^{(2)}(\alpha-\varsigma)\right] \\
& =\int d^{2} \varsigma P_{\text {in }}(\varsigma) \cdot \frac{1}{\pi|\varsigma|^{2} / M} \exp \left(-\frac{|\alpha|^{2}}{|\varsigma|^{2} / M}\right) .
\end{aligned}
$$

First, we use Eq. (12) to obtain the high-order moments of the scattered light,

$$
\begin{aligned}
& \left\langle\hat{a}^{\dagger n} \hat{a}^{n}\right\rangle_{\text {out }}=\int d^{2} \alpha|\alpha|^{2 n} P_{\text {out }}(\alpha) \\
& =\int d^{2} \varsigma \int d^{2} \alpha \frac{M}{\pi|\varsigma|^{2}} e^{-\frac{M}{|\varsigma|^{2}}|\alpha|^{2}}|\alpha|^{2 n} \cdot P_{\text {in }}(\varsigma) \\
& =\int d^{2} \varsigma P_{\text {in }}(\varsigma) \cdot \frac{|\varsigma|^{2 n}}{M^{n}} n !=\left\langle\hat{a}^{\dagger n} \hat{a}^{n}\right\rangle_{\text {in }} \cdot \frac{n !}{M^{n}}
\end{aligned}
$$

Therefore, for arbitrary input states, the $n$-order correlation function of the scattered light is

$$
g_{\text {out }}^{(n)}=\frac{\left\langle\hat{a}^{\dagger n} \hat{a}^{n}\right\rangle_{\text {out }}}{\left(\left\langle\hat{a}^{\dagger} \hat{a}\right\rangle_{\text {out }}\right)^{n}}=\frac{n !\left\langle\hat{a}^{\dagger n} \hat{a}^{n}\right\rangle_{\text {in }} / M^{n}}{\left(\left\langle\hat{a}^{\dagger} \hat{a}\right\rangle_{\text {in }} / M\right)^{n}}=n ! g_{\text {in }}^{(n)} .
$$

This result holds for arbitrary input statistics. In particular, the 2 nd order gives $g_{\text {out }}^{(2)}=2 g_{\text {in }}^{(2)}$, which is just the result (9) in the many-diffuser limit $M \rightarrow \infty$. After the hierarchy scattering through $k$ RGGs, the final output light gives $g_{\text {out }-k}^{(n)} \simeq(n !)^{k} g_{\text {in }}^{(n)}$.

Next we use the output $P$-function (12) to obtain the photon statistics, and compare it with the above result (2) based on combination counting. Since the scattered state can be written as $\hat{\rho}_{\text {out }}=\int d^{2} \alpha P_{\text {out }}(\alpha)|\alpha\rangle\left\langle\alpha\left|=\sum \tilde{P}_{n}\right| n\right\rangle\langle n|$, the photon 


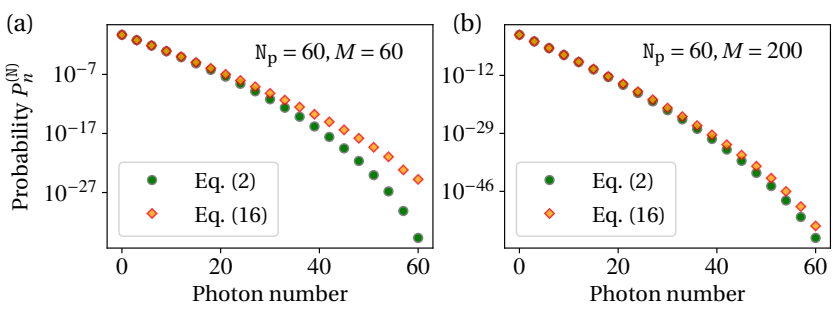

Figure 4. The scattered distribution $P_{n}$ from Fock input $\left(\mathrm{N}_{\mathrm{P}}=60\right)$ calculated from Eq. (2) (based on combination counting) and Eq. (16) [based on the mapping relation (11)] for (a) $M=60$ (b) $M=200$. For large $M$, they almost coincide with each other.

number distribution $\tilde{P}_{n}$ also can be obtained as

$$
\begin{aligned}
\tilde{P}_{n} & =\left\langle n\left|\hat{\rho}_{\text {out }}\right| n\right\rangle=\int d^{2} \alpha P_{\text {out }}(\alpha) \cdot \frac{|\alpha|^{2 n}}{n !} e^{-|\alpha|^{2}} \\
& =\int d^{2} \varsigma P_{\text {in }}(\varsigma) \frac{M /|\varsigma|^{2}}{\left(1+M /|\varsigma|^{2}\right)^{n+1}} .
\end{aligned}
$$

For a Fock input state $|\mathrm{N}\rangle$, the input $P$-function is $P_{\text {in }}(\varsigma)=$ $e^{|\varsigma|^{2}}\left[\partial_{\varsigma}^{\mathbb{N}} \partial_{\varsigma^{*}}^{\mathbb{N}} \delta^{(2)}(\varsigma)\right] / \mathrm{N} ![4]$, and the above integral gives

$$
\begin{aligned}
\tilde{P}_{n}^{(\mathrm{N})} & =\lim _{\varsigma \rightarrow 0} \frac{1}{\mathrm{~N} !} \partial_{\varsigma}^{\mathrm{N}} \partial_{\varsigma^{*}}^{\mathrm{N}}\left[\frac{e^{|\varsigma|^{2}} M /|\varsigma|^{2}}{\left(1+M /|\varsigma|^{2}\right)^{n+1}}\right] \\
& =\frac{\mathrm{N} !}{n !} \sum_{k=n}^{\mathrm{N}} \frac{(-1)^{k-n} k !}{(\mathrm{N}-k) !(k-n) ! M^{k}}
\end{aligned}
$$

and $\tilde{P}_{n}^{(\mathrm{N})}=0$ for $n>\mathrm{N}$ (see Appendix A).

Here we emphasize Eqs. $(15,16)$ are valid only in the manydiffuser limit $M \rightarrow \infty$, which guarantees the Poisson input must exactly produce the thermal state and $g_{\text {out }}^{(2)}=2 g_{\text {in }}^{(2)}$ holds exactly. Thus generally $\tilde{P}_{n}^{(\mathrm{N})}$ is not equivalent with the result (2) based on combination counting, which applies in more general cases. When $M$ is large, their difference becomes negligible, especially in the few-photon regime (Fig. 4).

\section{SUMMARY}

In this paper, we develop a quantum framework to study the full photon number statistics of the scattered light passing through the RGG. The output statistics is obtained by counting the combinations how the input photons are distributed on the scattered light pattern. When the total photon number of the whole scattered light pattern is approximately a constant, counting the photon number received by a small area on the light pattern naturally gives the thermal statistics. Then for arbitrary input states, the scattered photon statistics also can be well obtained by taking the input statistics into account.

We also obtain the $P$-function of the scattered light from arbitrary input states in the many-diffuser limit. With the help of these distributions, we find that the scattered light always gives a relation $g_{\text {out }}^{(n)} \simeq n ! g_{\text {in }}^{(n)}$, which holds for any order- $n$ and any input state, including non-classical ones. In these situations, this theory provides a more precise description beyond the previous classical theory based on central limit theorem.

The above results of $g^{(n)}$-correlations scattered from nonclassical input can be verified by squeezed light in current experiments, and may be utilized for correlation imaging of high orders. This result also indicates it is possible to create a scattered light with very high $g^{(n)}$-correlations simply, which may be utilized to enhance the multi-photon absorption processes and high harmonic generation [39-41].

Acknowledgment - SWL appreciates very much for the helpful discussion with R. Nessler in Texas A\&M University, W.-K. Yu in Beijing Institute of Technology, and J. Sperling in Universität Rostock. This study is supported by NSF of China (Grant No. 11905007), Beijing Institute of Technology Research Fund Program for Young Scholars, AFOSR award FA9550-18-1-0141, ONR award N00014-16-1-3054, and Robert A. Welch Foundation award A-1261.

\section{Appendix A: Calculation of Eq. (16)}

The $P$-function of the Fock state $|\mathrm{N}\rangle$ is a highly singular function $P_{\text {in }}(\varsigma)=e^{|\varsigma|^{2}}\left[\partial_{\varsigma}^{\mathrm{N}} \partial_{\varsigma^{*}}^{\mathrm{N}} \delta^{(2)}(\varsigma)\right] / \mathrm{N} !$, which contains the derivative of $\delta$-function. Notice that for $\varsigma=\varsigma_{x}+i \varsigma_{y}$, the derivative gives $\partial_{\varsigma} \partial_{\varsigma^{*}}=\frac{1}{4}\left(\partial_{\varsigma_{x}}^{2}+\partial_{\varsigma_{y}}^{2}\right)=\frac{1}{4} \nabla^{2}$. From the Stokes theorem we obtain $\int d^{2} \varsigma\left[f(\varsigma) \nabla^{2 n} \delta^{(2)}(\varsigma)-\delta^{(2)}(\varsigma) \nabla^{2 n} f(\varsigma)\right]=0$, thus the integral (15) gives

$$
\begin{aligned}
\tilde{P}_{n} & =\frac{1}{\mathrm{~N} !} \int d^{2} \varsigma\left[\frac{\partial^{2 \mathrm{~N}}}{\partial \varsigma^{\mathrm{N}} \partial \varsigma^{* \mathrm{~N}}} \delta^{(2)}(\varsigma)\right] e^{|\varsigma|^{2}} \frac{M /|\varsigma|^{2}}{\left(1+M /|\varsigma|^{2}\right)^{n+1}}=\frac{1}{\mathrm{~N} !} \int d^{2} \varsigma \delta^{(2)}(\varsigma) \frac{\partial^{2 \mathrm{~N}}}{\partial \varsigma^{\mathrm{N}} \partial \varsigma^{* \mathrm{~N}}} \frac{e^{|\varsigma|^{2}} M /|\varsigma|^{2}}{\left(1+M /|\varsigma|^{2}\right)^{n+1}} \\
& =\lim _{\varsigma \rightarrow 0} \frac{1}{\mathrm{~N} !} \partial_{\varsigma}^{\mathrm{N}} \partial_{\varsigma^{*}}^{\mathrm{N}}\left[\frac{e^{|\varsigma|^{2}} M /|\varsigma|^{2}}{\left(1+M /|\varsigma|^{2}\right)^{n+1}}\right] .
\end{aligned}
$$

Now we need to calculate this derivative and taking the limit. Denoting $\varsigma \rightarrow x, \varsigma^{*} \rightarrow y$, the above derivative gives 


$$
\begin{aligned}
\partial_{y}^{\mathrm{N}}\left[\frac{e^{x y} M(x y)^{n}}{(M+x y)^{n+1}}\right] & =\sum_{k=0}^{\mathrm{N}} C_{\mathrm{N}}^{k} \cdot \partial_{y}^{\mathrm{N}-k} e^{x y} \cdot \partial_{y}^{k} \frac{M(x y)^{n}}{(M+x y)^{n+1}}=\sum_{k=0}^{\mathrm{N}} C_{\mathrm{N}}^{k} \cdot e^{x y} x^{\mathrm{N}-k} \cdot \sum_{q=0}^{k} C_{k}^{q} \cdot \partial_{y}^{q}\left[M(x y)^{n}\right] \cdot \partial_{y}^{k-q} \frac{1}{[M+x y]^{n+1}} \\
& =\sum_{k=0}^{\mathrm{N}} \sum_{q=0}^{k} C_{\mathrm{N}}^{k} C_{k}^{q} \cdot e^{x y} x^{\mathrm{N}-k} \cdot M x^{n} \frac{n !}{(n-q) !} y^{n-q} \cdot(-1)^{k-q} \frac{(n+k-q) !}{n !}(M+x y)^{-n-1-k+q} x^{k-q} \\
& =M \sum_{k=0}^{\mathrm{N}} \sum_{q=0}^{k} \frac{(-1)^{k-q}(n+k-q) !}{(n-q) !} C_{\mathrm{N}}^{k} C_{k}^{q}\left[y^{n-q} \cdot x^{\mathrm{N}-q+n} e^{x y}(M+x y)^{-n-1-k+q}\right] .
\end{aligned}
$$

Now we need to further calculate the derivative $\partial_{x}^{\mathbb{N}}$ by applying the Leibniz rule to the above bracket, which gives $\sum_{l=0}^{\mathrm{N}} C_{\mathrm{N}}^{l}$. $\left[\partial_{x}^{l} x^{\mathbb{N}-q+n}\right] \cdot \partial_{x}^{\mathbb{N}-l}\left[e^{x y}(M+x y)^{-n-1-k+q}\right]$.

Since at last we need to take the limit $x, y \rightarrow 0$, from the power factor $\partial_{x}^{l} x^{\mathrm{N}-q+n}=\frac{(\mathrm{N}-q+n) !}{(\mathrm{N}-q+n-l) !} x^{\mathrm{N}-q+n-l}$ we can notice that only few terms in the above summations could exist, and they must satisfy $\mathrm{N}-q+n-l=0$, thus we have $q-n=\mathrm{N}-l \geq 0$. But since the denominator of Eq. (A2) contains $(n-q)$ ! which gives $+\infty$ for $n<q$, that guarantees $q=n$ and $l=\mathrm{N}$. Therefore, taking the limit of the above derivative gives

$$
\lim _{x, y \rightarrow 0} \partial_{x}^{\mathbb{N}} \partial_{y}^{\mathbb{N}}\left[\frac{e^{x y} M(x y)^{n}}{(M+x y)^{n+1}}\right]=\sum_{k=0}^{\mathbb{N}}(-1)^{k-n} k ! \cdot \frac{\mathrm{N} !}{(\mathrm{N}-k) ! k !} \cdot \frac{k !}{(k-n) ! n !} \cdot \frac{\mathrm{N} !}{M^{k}},
$$

as shown in the main text.

[1] M. Scully and W. E. Lamb, Phys. Rev. Lett. 16, 853 (1966).

[2] M. O. Scully and W. E. Lamb, Phys. Rev. 159, 208 (1967).

[3] M. O. Scully and M. S. Zubairy, Quantum optics (Cambridge university press, 1997).

[4] G. S. Agarwal, Quantum Optics, 1st ed. (Cambridge University Press, Cambridge, UK, 2012).

[5] W. Martienssen and E. Spiller, Am. J. Phys. 32, 919 (1964).

[6] F. T. Arecchi, Phys. Rev. Lett. 15, 912 (1965).

[7] F. Arecchi, A. Berne, A. Sona, and P. Burlamacchi, IEEE J. Quant. Electr. 2, 341 (1966).

[8] F. T. Arecchi, A. Berné, and P. Bulamacchi, Phys. Rev. Lett. 16, 32 (1966).

[9] L. E. Estes, L. M. Narducci, and R. A. Tuft, J. Opt. Soc. Am. 61, 1301 (1971).

[10] P. R. Pearl and G. J. Troup, Opto-electronics 1, 152 (1969).

[11] A. Gatti, E. Brambilla, M. Bache, and L. A. Lugiato, Phys. Rev. Lett. 93, 093602 (2004).

[12] A. Valencia, G. Scarcelli, M. D'Angelo, and Y. Shih, Phys. Rev. Lett. 94, 063601 (2005).

[13] F. Ferri, D. Magatti, A. Gatti, M. Bache, E. Brambilla, and L. A. Lugiato, Phys. Rev. Lett. 94, 183602 (2005).

[14] D.-Z. Cao, G.-J. Ge, and K. Wang, Appl. Phys. Lett. 97, 051105 (2010).

[15] F. Li, C. Altuzarra, t. Li, M. O. Scully, and G. S. Agarwal, J. Opt. (2019), 10.1088/2040-8986/ab48d4.

[16] T. Peng, H. Chen, Y. Shih, and M. O. Scully, Phys. Rev. Lett. 112, 180401 (2014).

[17] T. Peng, J. Simon, H. Chen, R. French, and Y. Shih, Europhys. Lett. 109, 14003 (2015).

[18] Y. S. Ihn, Y. Kim, V. Tamma, and Y.-H. Kim, Phys. Rev. Lett. 119, 263603 (2017).

[19] J. W. Goodman, Statistical Optics, 1st ed. (Wiley-Interscience,
New York, 2000).

[20] J. W. Goodman, Speckle Phenomena in Optics, 1st ed. (W. H. Freeman, Englewood, Colo, 2010).

[21] L. Mandel, Proc. Phys. Soc. 72, 1037 (1958).

[22] L. Mandel, Proc. Phys. Soc. 74, 233 (1959).

[23] T. Mehringer, S. Mährlein, J. von Zanthier, and G. S. Agarwal, Opt. Lett. 43, 2304 (2018).

[24] N. Bender, H. Y1lmaz, Y. Bromberg, and H. Cao, Optica 5, 595 (2018).

[25] N. Bender, H. Yilmaz, Y. Bromberg, and H. Cao, Opt. Express 27, 6057 (2019).

[26] J. H. Shapiro and R. W. Boyd, Quant. Info. Proc. 11, 949 (2012).

[27] J. Sperling, W. Vogel, and G. S. Agarwal, Phys. Rev. Lett. 109, 093601 (2012).

[28] J. Sperling, W. Vogel, and G. S. Agarwal, Phys. Rev. A 85, 023820 (2012).

[29] L. A. Jiang, E. A. Dauler, and J. T. Chang, Phys. Rev. A 75, 062325 (2007).

[30] B. E. Kardynał, Z. L. Yuan, and A. J. Shields, Nature Photonics 2, 425 (2008).

[31] L. Landau and E. Lifshitz, Statistical Physics, Part 1 (Butterworth-Heinemann, Oxford, 1980).

[32] K. Huang, Statistical Mechanics, 2nd ed. (Wiley, New York, 1987).

[33] D. Z. Xu, S.-W. Li, X. F. Liu, and C. P. Sun, Phys. Rev. E 90, 062125 (2014).

[34] H. Dong, S. Yang, X. F. Liu, and C. P. Sun, Phys. Rev. A 76, 044104 (2007).

[35] R. Liu, A. Fang, Y. Zhou, P. Zhang, S. Gao, H. Li, H. Gao, and F. Li, Phys. Rev. A 93, 013822 (2016).

[36] C. C. Gerry and P. Knight, Introductory quantum optics (Cam- 
bridge University Press, Cambridge, UK ; New York, 2005).

[37] Y. Zhou, F.-1. Li, B. Bai, H. Chen, J. Liu, Z. Xu, and H. Zheng, Phys. Rev. A 95, 053809 (2017).

[38] G. S. Agarwal and K. Tara, Phys. Rev. A 46, 485 (1992).

[39] K. Y. Spasibko, D. A. Kopylov, V. L. Krutyanskiy, T. V. Murz- ina, G. Leuchs, and M. V. Chekhova, Phys. Rev. Lett. 119 , 223603 (2017).

[40] G. S. Agarwal, Phys. Rev. A 1, 1445 (1970).

[41] A. Jechow, M. Seefeldt, H. Kurzke, A. Heuer, and R. Menzel, Nature Photon. 7, 973 (2013). 